Abstract P5-S6.19 Table 1 Prevalence of STls and asymptomatic infections $(\mathrm{n}=282)$

\begin{tabular}{llll}
\hline Pathogen & Baseline visit & 1st follow-up & Final visit \\
\hline Cervical & $27.7 \%$ (asymptomatic & 16.7\% (asymptomatic & $21.3 \%$ (asymptomatic \\
infections & $35.9 \%$ ) & $60.5 \%$ ) & $76.7 \%$ ) \\
(GC and/or CT) & & & \\
TV & $29.9 \%$ (asymptomatic & & $46.6 \%$ (asymptomatic \\
& $27.2 \%$ ) & & $82.2 \%)$ \\
Latent & $6.8 \%$ & $2.5 \%$ \\
syphilis (RPR & & \\
titre $>1: 8$ ) & & \\
\hline
\end{tabular}

Conclusions The incidence of GC, CT and TV and the considerable burden of asymptomatic infections indicate the need for periodic presumptive treatment at high STI prevalence sites in India. The current regime of bi-annual syphilis screening is justified. Consistent condom use and partner treatment, particularly for regular partners, need to be re-emphasised.

\section{P5-S6.20 STIS AND HEALTHCARE REFORM. WILL IT REALLY IMPROVE AMERICA'S SEXUAL HEALTH?}

doi:10.1136/sextrans-2011-050108.576

C Mettenbrink, R Cornelis. Denver Public Health Department,Denver USA

Background The implementation of healthcare reform in the U.S has led to a debate on whether publicly-funded STI clinics will continue to be necessary. A large proportion of STIs are reported from non-STI clinic providers and this proportion will grow with universal access to care. However, STI clinics serve vulnerable populations such as men who have sex with men (MSM) and racial/ ethnic minorities who have no other access to STI care. In addition, many prefer the confidentiality and expertise of STI clinics. We studied characteristics of patients reported with gonorrhoea in the City and County of Denver and compared those reported from the Denver Metro Health (STI) clinic (DMHC) with those reported from elsewhere.

Methods As part of the CDC-funded STD Surveillance Network (SSuN), DMHC and the Colorado Department of Public Health and Environment (CDPHE) collect additional risk information for gonorrhoea cases residing in the SSuN catchment area. Of all incident gonorrhoea cases within a given month, 40 are randomly selected for an interview by CDPHE staff to collect demographic, treatment, and risk information. All gonorrhoea cases diagnosed between 1 January 2008 and 31 October 2010 were examined.

Results Of 2933 gonorrhoea cases for the 2-year period, 932 (32\%) were diagnosed at DMHC. Cases diagnosed at DMHC were considerably more likely to be male $(74 \%$ vs $38 \% \mathrm{p}<0.0001)$, and tended to be slightly older (mean age 27.0 vs $24.9, p=<0.0001$ ) than cases diagnosed elsewhere. CDPHE successfully contacted $41 \%$ of selected cases for interviews. A significantly higher proportion of cases diagnosed in the STI clinic were MSM compared to cases diagnosed elsewhere $(34.7 \%$ vs $14.5 \%, \mathrm{p} \leq 0.0001)$. No differences were found in terms of race/ethnicity, number of reported sex partners, education level, or previous gonorrhoea infection.

Conclusions In the City and County of Denver, heterosexual men and MSM are more likely to be diagnosed with gonorrhoea in the STI clinic than women. The successful transition of STI services to non-STI healthcare systems in the context of healthcare reform, will not only depend on the STI expertise in those settings, but in large part on a shift in health seeking behaviours among men, many of whom may be considered core STI transmitters. The continued need for and utilisation of STI clinics in countries with long-standing universal access to care, should give us pause in abandoning our STI clinical system to soon.

\section{P5-S6.21 ACYCLOVIR FOR MANAGING GENITAL ULCER DISEASE IN SOUTH AFRICA: EXPERIENCES OF GUIDELINE INTRODUCTION, IMPLEMENTATION AND UPTAKE}

doi:10.1136/sextrans-2011-050108.577

${ }^{1} \mathrm{D}$ Emge, ${ }^{2} \mathrm{~S}$ Delany-Moretlwe, ${ }^{3} \mathrm{P}$ Mayaud. 'London School of Hygiene \& Tropical Medicine and Reproductive Health \& HIV Research Unit, University of the Witwatersrand, Bay City, USA; ${ }^{2}$ Reproductive Health \& HIV Research Unit, University of the Witwatersrand, Hillbrow, Johannesburg, South Africa; ${ }^{3}$ London School of Hygiene $g$ Tropical Medicine, London, UK

Background Herpes simplex virus type 2 (HSV-2) is the leading cause of genital ulcer disease (GUD) worldwide. Treatment with acyclovir has been shown to shorten GUD duration, decrease plasma and genital HIV RNA levels and slow HIV disease progression in several trials. In South Africa, STI management guidelines were revised in 2008 to include acyclovir as first-line GUD therapy. However, acyclovir access appeared to be unequal across various settings. This study attempted to understand acyclovir access in South Africa from both a supply and demand perspective.

Methods The study was cross-sectional and primarily qualitative. Three groups of participants were recruited to reflect acyclovir access and use: policymaker key informants, healthcare providers at public primary healthcare facilities and ART initiation sites in greater Johannesburg, and former HSV-2 treatment trial participants (both genders, HIV-positive and -negative). Information was collected following the WHO/Health Action International twostage cluster random selection of health units through standardised questionnaires, complemented by in-depth interviews with selected stakeholders.

Results Acyclovir was documented to be widely available. Challenges to access included the initial policy development and implementation process, staff training, accessibility and availability. The updated guidelines appeared to have been introduced with little high-level co-ordination and minimal staff training, although policymakers and staff thought that HSV-2 treatment was both efficacious and useful. Clinics did not experience drug stock outs. The demand for acyclovir appeared to be poor and influenced by limited knowledge of HSV-2 by the general public, negative perceptions associated with HSV-2 infection, adverse logistical factors like long waiting times at clinics and negative experiences with staff at clinics.

Conclusions The South African guidelines were supported by local research evidence in line with WHO recommendations. Although little attention was paid to how the new guidelines had to be introduced, implemented and monitored, both the supply and prescribers' awareness improved rapidly. Access to acyclovir was undermined by poor demand from patients. Individuals possibly did not see herpes as enough of a problem to seek treatment. To ensure the success of new treatment approaches, it is essential to consider user and well as provider issues.

\section{P5-S6.22 SOCIAL-STRUCTURAL FACTORS ASSOCIATED WITH SUPPORTIVE SERVICE USE IN A COHORT OF HIV-POSITIVE INDIVIDUALS ON ARV THERAPY IN BRITISH COLUMBIA, CANADA}

doi:10.1136/sextrans-2011-050108.578

${ }^{1} \mathrm{~N}$ O'Brien, ${ }^{1} \mathrm{~A}$ K Palmer, ${ }^{1} \mathrm{~W}$ Zhang, ${ }^{1} \mathrm{~W}$ Michelow, ${ }^{1} \mathrm{~A}$ Shen, ${ }^{2} \mathrm{E}$ Roth, ${ }^{3} \mathrm{C} \mathrm{L}$ Rhodes, ${ }^{1} \mathrm{~J} S \mathrm{G}$ Montaner, ${ }^{1} \mathrm{R}$ S Hogg. ${ }^{1} B C$-Centre for Excellence in HIVIAIDS, Vancouver, Canada; ${ }^{2}$ University of Victoria, Canada; ${ }^{3}$ University of Waterloo, Canada

Introduction Medical services are seldom the only assistance required for HIV-positive individuals to lead longer, healthier lives. The clinical complexities of HIV infection and the multiple needs of people living with HIV often require additional assistance to ensure 